\title{
AN ABELIAN THEOREM FOR A CLASS OF SUBHARMONIC FUNCTIONS
}

\author{
FARUK F. ABI-KHUZAM
}

\begin{abstract}
We show that if the Riesz-mass of a subharmonic function $u$, of finite order $\lambda$, is distributed along a ray, then regular variation (with exponent $\lambda$ ) of the mean value of $u\left(r e^{i \theta}\right)$ implies regular variation (with exponent $\lambda$ ) of each of the $L_{s}(-\pi, \pi)$ means of $u\left(r e^{i \theta}\right)$. This result extends a known theorem of Edrei and Fuchs, but our method differs from theirs. In particular, for the case of integral orders we obtain the theorem for a much more general distribution of the Riesz-mass. A corollary, which appears to be new, on the deficiency of the value zero of entire functions with positive integral order, follows.
\end{abstract}

Let $f$ be an entire function with real negative zeros and nonintegral order $\lambda$. Let $n(t)=n(t, 0)$ be the number of zeros of $f$ in $|z| \leqslant t$ and put

$$
N(r)=N(r, 0)=\int_{0}^{r} n(t) t^{-1} d t \quad(f(0) \neq 0) .
$$

Edrei and Fuchs [1, p. 340] have shown that the condition

$$
N(r) \sim r^{\lambda} L(r) \quad(r \rightarrow \infty, 0<\lambda<1)
$$

implies

$$
\begin{aligned}
T(r) & \sim r^{\lambda} L(r) \quad \text { if } 0<\lambda \leqslant \frac{1}{2} \\
& \sim(\csc \pi \lambda) r^{\lambda} L(r) \quad \text { if } \frac{1}{2}<\lambda<1 .
\end{aligned}
$$

Here

$$
T(r)=T(r, f)=\frac{1}{2 \pi} \int_{-\pi}^{\pi} \log ^{+}\left|f\left(r e^{i \theta}\right)\right| d \theta
$$

is the Nevanlinna characteristic of $f$, and $L$ is a slowly varying function, i.e. $L$ is positive and satisfies

$$
\lim _{r \rightarrow \infty} \frac{L(a r)}{L(r)}=1
$$

for every $a>0$.

In this note we obtain an analogue of the above result of Edrei and Fuchs in which:

(i) $\log |f|$ is replaced by (a) a subharmonic function $u$ whose Riesz mass is

Received by the editors March 11, 1976.

AMS (MOS) subject classifications (1970). Primary 30A64, 31A10; Secondary 30A68.

Key words and phrases. Riesz-mass, order of a subharmonic function, regular variation. 
distributed along the negative $x$-axis, and whose order $\lambda$ is finite and nonintegral, or (b) a subharmonic function $u$ whose order $\lambda$ is a positive integer and whose Riesz mass is concentrated in the set $D(\eta, \varphi)=\bigcup_{j=0}^{\lambda}\{z$ : $|\arg z-v-2 \pi j / \lambda| \leqslant \eta\}$ for some $\varphi \in[0 ; 2 \pi)$ and $\eta \in[0, \pi / 2 \lambda)$.

(ii) $T(r)$ is replaced by any $m_{s}(r, u)$ where

$$
m_{s}(r, u)=\left\{(2 \pi)^{-1} \int_{-\pi}^{\pi}\left|u\left(r e^{i \theta}\right)\right|^{s} d \theta\right\}^{1 / s} \quad(1<s<\infty) .
$$

As a corollary, we obtain a result about the deficiency of the value zero of a class of entire functions with positive integral order; in addition, our proof may be of interest because of its simplicity.

Let $u$ be a function subharmonic in the plane and harmonic in a neighbourhood of the origin. Put $u^{+}=\max (u, 0)$ and assume for convenience that $u(0)=0$. The characteristic of $u$ is defined by

$$
T(r)=T(r, u)=(2 \pi)^{-1} \int_{-\pi}^{\pi} u^{+}\left(r e^{i \theta}\right) d \theta,
$$

and the order $\lambda$ of $u$ is defined by

$$
\lambda=\limsup _{r \rightarrow \infty} \frac{\log T(r)}{\log r} .
$$

The customary way of defining $N$ is to let $n(t)=n(t, u)=\int_{|a|<r} d \mu(a)$, where $\mu$ is the positive mass-distribution associated to $u$ via the Riesz Decomposition Theorem, and then to put $N(r)=N(r, u)=\int_{0}^{r} n(t) t^{-1} d t$. If $u$ is harmonic in a neighbourhood of the origin with $u(0)=0$ then $\int_{0}^{r} n(t) t^{-1} d t$ exists and

$$
N(r)=N(r, u)=(2 \pi)^{-1} \int_{-\pi}^{\pi} u\left(r e^{i \theta}\right) d \theta .
$$

For $\lambda$ a positive integer, let $\psi_{\lambda}(\theta)=\cos \lambda \theta$. For nonintegral $\lambda$ let $\psi_{\lambda}(\theta)=$ $\pi \lambda \csc \pi \lambda \cos \lambda \theta$ and let $m_{s}\left(\psi_{\lambda}\right)$ be the $L_{s}(-\pi, \pi)$ norm of $\psi_{\lambda}$. We are now ready to state our result:

THEOREM. Let $u$ be a function subharmonic in the plane, having finite order $\lambda$ with $u(z)=\operatorname{Re} \sum_{m=1}^{\infty} \alpha_{m} z^{m}$ for $z$ near zero and $q=[\lambda]$. Assume that as $r \rightarrow \infty$

$$
N(r)=N(r, u) \sim r^{\lambda} L(r)
$$

where $L$ is a slowly varying function.

(I) If the order $\lambda$ of $u$ is not an integer and if the Riesz mass of $u$ is distributed along the negative $x$-axis. then

$$
\begin{array}{ll}
m_{s}(r, u) \sim m_{s}\left(\psi_{\lambda}\right) r^{\lambda} L(r) & (1 \leqslant s<\infty), \\
m_{s}\left(r, u^{+}\right) \sim m_{s}\left(\psi_{\lambda}^{+}\right) r^{\lambda} L(r) & (1 \leqslant s<\infty) .
\end{array}
$$

(II) Put

$$
c(r)=\alpha_{q}+\frac{1}{q} \int_{|a|<r} a^{-q} d \mu(a) .
$$


If the order $\lambda$ of $u$ is a positive integer and if the Riesz mass of $u$ is concentrated in a set

$$
D(\eta, \varphi)=\bigcup_{j=0}^{\lambda}\{z:|\arg z-\varphi-2 \pi j / \lambda|<\eta\}
$$

for some $\varphi \in[0,2 \pi)$ and $\eta \in[0, \pi / 2 \lambda)$, then $|c(r)|$ is a slowly varying function and

$$
m_{s}\left(r, u^{+}\right) \sim \frac{1}{2} m_{s}(r, u) \sim \frac{1}{2} m_{s}\left(\psi_{\lambda}\right) r^{q}|c(r)| \quad(1<s<\infty) .
$$

Proof of (I). Denote by $\left\{\gamma_{m}(r)\right\}$ the Fourier coefficients of $u$, i.e.

$$
\gamma_{m}(r)=(2 \pi)^{-1} \int_{-\pi}^{\pi} u\left(r e^{i \theta}\right) e^{-i m \theta} d \theta
$$

If $q=[\lambda]$ and $m>q+1$, then by the analogue of Hadamard's Theorem for subharmonic functions of finite order, we have, as in [3, p. 380],

$$
(-1)^{m} \gamma_{m}(r)=N(r)-\frac{m}{2}\left\{\int_{0}^{r}\left(\frac{t}{r}\right)^{m} N(t) \frac{d t}{t}+\int_{r}^{\infty}\left(\frac{r}{t}\right)^{m} N(t) \frac{d t}{t}\right\}
$$

Similarly, when $q \neq 0$ and $1<m<q$ we have

$$
(-1)^{m} \gamma_{m}(r)=\frac{1}{2} \alpha_{m} r^{m}+\frac{1}{2} m \int_{0}^{r}\left\{\left(\frac{r}{t}\right)^{m}-\left(\frac{t}{r}\right)^{m}\right\} N(t) \frac{d t}{t}+N(r)
$$

Clearly, $\gamma_{-m}(r)=\gamma_{m}(r)(m>0)$ and $\gamma_{0}(r)=N(r)$ by (7).

(8) implies [2, p. 273]

$$
\frac{r^{m+1} N(r)}{\int_{0}^{r} t^{m} N(t) d t} \rightarrow m+\lambda+1 \quad(m+\lambda+1>0, r \rightarrow \infty)
$$

and

$$
\frac{r^{m+1} N(r)}{\int_{r}^{\infty} t^{m} N(t) d t} \rightarrow|m+\lambda+1| \quad(m+\lambda+1<0, r \rightarrow \infty)
$$

Furthermore, since $L$ is slowly varying $r^{-a} L(r) \rightarrow 0$ for every $a>0$. In particular, since $q=[\lambda]$ and $N(r) \sim r^{\lambda} L(r)$,

$$
r^{m}=o(N(r)) \quad(1 \leqslant m \leqslant q, q \neq 0, r \rightarrow \infty) .
$$

Using (15), (16) and (17) in (13) and (14), a straightforward computation gives

$$
\gamma_{m}(r) / N(r) \rightarrow(-1)^{m} \lambda^{2} /\left(\lambda^{2}-m^{2}\right) \quad(r \rightarrow \infty, \text { all } m) .
$$

Suppose now that $1 \leqslant s \leqslant 2$. Using the fact that the $L_{s}$-norm is a nondecreasing function of $s$ together with Parseval's identity and the identity

$$
\pi \lambda \csc \pi \lambda \cos \lambda \theta=\sum_{-\infty}^{\infty} \frac{(-1)^{m} \lambda^{2}}{\lambda^{2}-m^{2}} e^{i m \theta}
$$

we have, in view of (18), 


$$
\begin{aligned}
\varlimsup_{r \rightarrow \infty}\left\{(2 \pi)^{-1}\right. & \left.\int_{-\pi}^{\pi}\left|\frac{u\left(r e^{i \theta}\right)}{N(r)}-\psi_{\lambda}(\theta)\right|^{s} d \theta\right\}^{1 / s} \\
& <\varlimsup_{r \rightarrow \infty}\left\{\sum_{m=-\infty}^{\infty}\left|\frac{\gamma_{m}(r)}{N(r)}-\frac{(-1)^{m} \lambda^{2}}{\lambda^{2}-m^{2}}\right|^{2}\right\}^{1 / 2} \\
& =\left\{\sum_{m=-\infty}^{\infty} \lim _{r \rightarrow \infty}\left|\frac{\gamma_{m}(r)}{N(r)}-\frac{(-1)^{m} \lambda^{2}}{\lambda^{2}-m^{2}}\right|^{2}\right\}^{1 / 2}=0,
\end{aligned}
$$

provided that the passage to the limit inside the summation is justified. This is done below, after our discussion of Case (II).

Now the first formula of (9) follows from (19) by Minkowski's inequality. The second formula of (9) follows from $\int\left|u^{+} / N-\psi_{\lambda}^{+}\right|^{s}<\int\left|u / N-\psi_{\lambda}\right|^{s}$, (19) and Minkowski's inequality.

If $2<s<\infty$, we let $s^{\prime}$ be the index conjugate to $s$, i.e., $\left(1 / s+1 / s^{\prime}=1\right)$, and apply the Hausdorff-Young Theorem. This gives an inequality analogous to (19) except that the $l_{2}$-norm on the right-hand side of (19) will be replaced by the $l_{s}$-norm, and then (9) follows as in the case $1 \leqslant s<2$.

Proof of (II). In what follows we denote by $\left\{c_{m}(r)\right\}$ the Fourier coefficients of $u$, and we define $\gamma_{m}(r)$ for $m \geqslant q+1$ by (13) and (if $q \geqslant 1$ ) for $1<m<q$ by (14) with $\alpha_{m}$ replaced by $\left|\alpha_{m}\right|$. Then we have

$$
\left|c_{m}(r)\right| \leqslant \gamma_{m}(r) .
$$

Suppose now that $1 \leqslant s \leqslant 2$. Proceeding as in the Proof of $I$ and using (20) we obtain

$$
\begin{gathered}
\varlimsup_{r \rightarrow \infty}\left\{(2 \pi)^{-1} \int_{-\pi}^{\pi}\left|\frac{u\left(r e^{i \theta}\right)-\left(c(r) e^{i q \theta}+\overline{c(r)} e^{-i q \theta}\right) 2^{-1} r^{q}}{N(r)}\right|^{s} d \theta\right\}^{1 / s} \\
\leqslant \varlimsup_{r \rightarrow \infty}\left\{\sum_{|m| \neq q}\left|\frac{\gamma_{m}(r)}{N(r)}\right|^{2}+2\left|\frac{c_{q}(r)-\left(r^{q} / 2\right) c(r)}{N(r)}\right|^{2}\right\}^{1 / 2}<\infty .
\end{gathered}
$$

I claim that (8) and (11) imply that

$$
r^{-q} N(r)=o(|c(r)|) \quad \text { as } r \rightarrow \infty .
$$

Let us assume (22). Noting that (22) implies that $c(r) \neq 0$ for large $r$, and denoting by $\Theta(r)$ one of the determinations of the argument of $c(r)$, we have from (22) and (21), 


$$
\lim _{r \rightarrow \infty}\left\{(2 \pi)^{-1} \int_{-\pi}^{\pi}\left|\frac{u\left(r e^{i \theta}\right)}{r^{q}|c(r)|}-\cos q(\theta+\Theta(r))\right|^{s} d \theta\right\}^{1 / s}=0
$$

from which (12) follows for $1 \leqslant s \leqslant 2$.

The case $2<s<\infty$ is dealt with in exactly the same fashion as in (I).

Before proving (22) we show that it implies that $|c(r)|$ is a slowly varying function: Let $a>1$ be fixed. We have

$$
\begin{array}{r}
|c(a r)-c(r)|=\left|q^{-1} \int_{r<|Z|<a r} Z^{-q} d_{\mu}(Z)\right|=O\left(\left|q^{-1} \int_{r}^{a r} t^{-q} d n(t)\right|\right) \\
=O\left(r^{-q} n(a r)\right)=O\left(r^{-q} N(2 a r)\right)=O\left(r^{-q} N(r)\right)=o(|c(r)|)
\end{array}
$$

the constants implied in the $O$-notation depending on $q$ and $a$ only. This shows that $|c(r)|$ is a slowly varying function.

We now prove (22) and for this purpose we distinguish two cases:
(A) $\int_{0}^{\infty} t^{-q-1} N(t) d t<\infty$ and
(B) $\int_{0}^{\infty} t^{-q-1} N(t) d t=\infty$

If (A) holds, then

$$
\begin{gathered}
r^{-q} N(r) \rightarrow 0, \quad r^{-q} n(r) \rightarrow 0, \quad r^{-q} N(r) / \int_{r}^{\infty} t^{-q-1} N(t) d t \rightarrow 0 \\
r^{-q} n(r) / \int_{r}^{\infty} t^{-q-1} n(t) d t \rightarrow 0 \quad \text { as } r \rightarrow \infty
\end{gathered}
$$

The third implication is (16) with $m+\lambda+1=0$. It is true [2, p. 237] by (8) and (A). The fourth implication follows from the third since $n(r) \sim \lambda N(r)$. (A) also implies that $q \alpha_{q}=-\int_{|a|<\infty} a^{-q} d \mu(a)$ and so $c(r)=$ $-q^{-1} \int_{|a|>r} a^{-q} d \mu(a)$. Using this with (11) we obtain

$$
\begin{aligned}
|c(r)| & =\left|c(r) e^{i q \varphi}\right|=\left|q^{-1} \int_{|a|>r}\left(a e^{-i \varphi}\right)^{-q} d \mu(a)\right| \\
& \geqslant q^{-1} \cos \lambda \eta \int_{r}^{\infty} t^{-q} d n(t) \\
& =q^{-1} \cos \lambda \eta\left\{-r^{-q} n(r)-q r^{-q} N(r)+q^{2} \int_{r}^{\infty} t^{-q-1} N(t) d t\right\} \\
& =q \cos \lambda \eta \int_{r}^{\infty} t^{-q-1} N(t) d t\{1-o(1)\}
\end{aligned}
$$

Now (26) and (25)(iii) give (22).

If (B) holds, then

$$
\operatorname{Re}\left(\alpha_{q} e^{i q \varphi}\right)+q \cos \lambda \eta \int_{0}^{r} t^{-q-1} N(t) d t \sim q \cos \lambda \eta \int_{0}^{r} t^{-q-1} N(t) d t,
$$

and so, in view of (11), we have 


$$
\begin{aligned}
|c(r)| & >\operatorname{Re}\left(\alpha_{q} e^{i q \varphi}\right)+q \cos \lambda \eta \int_{0}^{r} t^{-q-1} N(t) d t \\
& \sim q \cos \lambda \eta \int_{0}^{r} t^{-q-1} N(t) d t,
\end{aligned}
$$

which, when combined with (15), gives (22).

It thus remains to justify the finiteness statement in (21) and the passage to the limit under the summation in the " $l_{2}$-norm" in (19) and in the " $l_{s}$-norm" when $s^{\prime}>1$. This is a consequence of the following.

LEMMA. If $N(r) \sim r^{\lambda} L(r)$, then there exist absolute constants $K$ and $r_{0}$ such that

$$
\left|\gamma_{m}(r)\right| / N(r)<K|m|^{-1} \quad\left(r \geqslant r_{0}, m \geqslant q+1, q=[\lambda]\right) .
$$

Proof. Choose $\eta>0$ such that $1+\eta<2^{q+1-\lambda}$ (possible since $\lambda<q+$ 1), and then determine $r_{1}$ (by (4)) so that $L(2 r) / L(r)<1+\eta\left(r>r_{1}\right)$. From this and condition (8) on $N(r)$ we infer the existence of a number $r_{2}$ such that

$$
N(2 r) / N(r) \leqslant 2^{\lambda}(1+\eta) \text { for } r \geqslant r_{2} \text {. }
$$

From $N(r)=\int_{0}^{r} n(t) t^{-1} d t$ we have

$$
N(2 r) \geqslant \int_{r}^{2 r} n(t) t^{-1} d t>n(r) \log 2
$$

since $n(t)$ is nondecreasing. An integration by parts in (13) followed by use of (30) and (29) and some trivial estimates gives

$$
\left|\gamma_{m}(r)\right|<r^{m}(2 \log 2)^{-1} \int_{r}^{\infty} t^{-m-1} N(t) d t .
$$

Following Rubel [4, p. 440] we have, from (31),

$$
\begin{aligned}
\left|\gamma_{m}(r)\right| & <(2 \log 2)^{-1} r^{m} \sum_{k=0}^{\infty} N\left(2^{k+2} r\right) \int_{2^{k} r}^{2^{k+1} r} t^{-m-1} d t \\
& <(2 \log 2)^{-1}\left(2^{\lambda}(1+\eta)\right)^{2} m^{-1} N(r) \sum_{k=0}^{\infty}\left(2^{-m} 2^{\lambda}(1+\eta)\right)^{k} \\
& =K m^{-1} N(r)
\end{aligned}
$$

$\left(m>q, r \geqslant r_{0}=\max \left(r_{1}, r_{2}\right)\right)$. This establishes the lemma.

Since $s^{\prime}>1$, the lemma shows that $\sum_{|m|>q}\left|\gamma_{m}(r) / N(r)\right|^{s^{\prime}}$ is uniformly convergent for $r \geqslant r_{0}$ and thus the passage to the limit under the summation sign in (19) is justified. This also shows that the sum on the right-hand side of (21) is finite provided that $\left(c_{q}(r)-2^{-1} r^{q} c(r)\right)=O(N(r))$; but this follows from

$$
\begin{aligned}
\left|c_{q}(r)-2^{-1} r^{q} c(r)\right| & \leqslant(2 q)^{-1} \int_{0}^{r} r^{-q} t^{q} d n(t)<(2 q)^{-1} n(r) \\
& \leqslant(2 q \log 2)^{-1} N(2 r)=O(N(r)) .
\end{aligned}
$$

COROLlaRY. Let $f$ be an entire function with positive integral order $\lambda$. If the zeros of $f$ belong to some set $D(\eta, \varphi)$ defined by (11) and satisfy (8), then the 
deficiency of the value 0 is 1 and so, $f$ has 0 and $\infty$ as the only deficient values.

Proof. Put $u=\log |f(z)|$; then by (12) with $s=1, T(r, u)$ $\sim \frac{1}{2} m_{1}\left(\psi_{\lambda}\right) r^{q}|c(r)|$, which, with (22), gives $N(r)=o(T(r))$, and the result follows.

We note the example $F(z)=\cos \left(z^{\lambda}\right), \lambda$ a positive integer, which shows that the condition $\eta<\pi / 2 \lambda$ is sharp, since, for this $F, 0$ is not a deficient value.

\section{REFERENCES}

1. A. Edrei and W. H. J. Fuchs, Tauberian theorems for a class of meromorphic functions with negative zeros and positive poles, Internat. Conf. in Function Theory, "Nauka", Moscow, 1966, pp. 339-358.

2. W. Feller, An introduction to probability theory and its applications, Vol. II, Wiley, New York, 1966.

3. J. Miles and D. Shea, An extremal problem in value-distribution theory, Quart. J. Math. Oxford Ser. (2) 24 (1973), 377-383.

4. L. Rubel, A Fourier series method for entire functions, Duke Math. J. 30 (1963), 437-442.

Department of Mathematics, Cornell University, Ithaca, New York 14853

Current address: Department of Mathematics, American University of Beirut, Beirut, Lebanon 